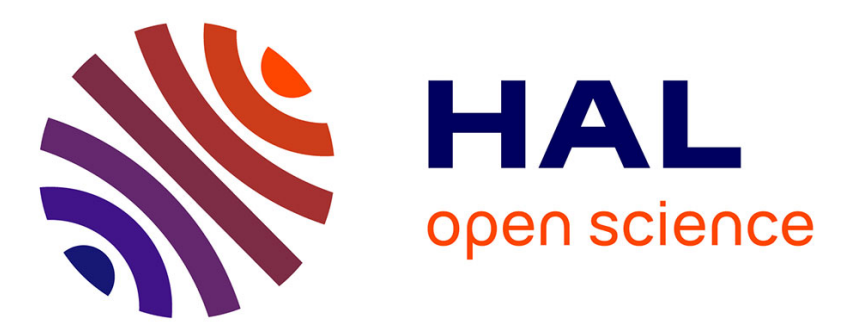

\title{
Improvement of transmission loss of a double panel by using active control with a virtual modal mass
}

\author{
Vincent Lhuillier, Simon Chesné, Luc Gaudiller, Charles Pezerat
}

\section{To cite this version:}

Vincent Lhuillier, Simon Chesné, Luc Gaudiller, Charles Pezerat. Improvement of transmission loss of a double panel by using active control with a virtual modal mass. Journal of Intelligent Material Systems and Structures, 2013, 24 (15), pp.1822-1833. 10.1177/1045389X13478269 . hal-00807817

\section{HAL Id: hal-00807817 https://hal.science/hal-00807817}

Submitted on 17 Mar 2016

HAL is a multi-disciplinary open access archive for the deposit and dissemination of scientific research documents, whether they are published or not. The documents may come from teaching and research institutions in France or abroad, or from public or private research centers.
L'archive ouverte pluridisciplinaire HAL, est destinée au dépôt et à la diffusion de documents scientifiques de niveau recherche, publiés ou non, émanant des établissements d'enseignement et de recherche français ou étrangers, des laboratoires publics ou privés. 
Author manuscript. Published in Journal of Intelligent Material Systems and Structures

24(15) 1822-1833; DOI :10.1177/1045389X13478269

\title{
Improvement of transmission loss of a double panel by using active control with a virtual modal mass
}

\author{
V. Lhuillier ${ }^{1 / 2 / 4}$, S. Chesné ${ }^{1}$, L. Gaudiller ${ }^{1}$, C. Pézerat ${ }^{3}$, \\ ${ }^{1}$ Université de Lyon, CNRS INSA-Lyon, LaMCoS UMR5259, F-69621, Villeurbanne, France. \\ 2 LVA, INSA-Lyon, F-69621, France. \\ ${ }^{3}$ LAUM, Université du Maine, CNRS, UMR 6613, F-72085 Le Mans, France. \\ ${ }^{4}$ LIGO Hanford Observatory, 127124 North Route 10, Richland, WA 99354, USA.
}

\section{Corresponding author : simon.chesne@insa-lyon.fr}

In this paper, modal feedback control is proposed to reduce the sound transmission through finite double panels, using PZT ceramic sensors and actuators bonded to the structure. Active control allows adding virtual modal damping and mass to the structure by the use of modal velocities and accelerations. In a first step, the equations describing the structure, the actuators, the acoustic excitation and the acoustic radiation are detailed. Next, the state space formulation of the smart structure is presented. In a second step, the implementation of active control is illustrated through the use of numerical examples. Finally, simulations are performed using 2 actuators, allowing 5 modes to be controlled. The transmission loss factors of the controlled and uncontrolled structure are shown as a function of the required command voltage. These results are also compared with those achieved using other vibroacoustic control techniques.

Key-words : Vibroacoustic control, Active control, Modal control, Sound Transmission, Smart structure 


\section{Introduction}

Double panels, made of two plates separated by an air cavity, are commonly employed when high transmission loss values must be achieved with lightweight structures. Although such panels can provide good sound insulation at mid-high frequencies, their performances decrease rapidly at low frequencies, especially in the vicinity of the massair-mass resonance frequency. Good passive isolation can be achieved through the use of cavity control or panel control. In recent years, considerable progress has been made in active noise control, thanks to the ever-increasing processing power of computers. In the case of cavity control, loudspeakers and error microphones are installed between the two panels, in order to modify the cavity pressure field and reduce the radiated sound power. $\mathrm{X}$-filtered LMS feed-forward control is often used in experimental research (Bao and Pan [1997]; Jakob et al. [2003a]; Sas et al. [2005]; Bouvet [1993]) due to its simplicity and the significant reduction in sound power which can be obtained. Nonetheless, measuring a reference signal under "real working conditions" is an obstacle to the development of this control approach : the non-availability of a reference signal limits feed-forward control. Although, feedback controllers have also been successfully implemented in Jakob et al. [2003b]; Kaiser et al. [2003] the feed-forward approach appears to be more efficient.

Prior to the development of active control, Mason and Fahy [1988] showed that a Helmholtz resonator, optimally tuned to the cavity resonance, increased the acoustic damping and allowed significant transmission loss enhancements. This approach can be adapted to a "passive control" technique using PZT and electric circuits. In an approach similar to that of a Helmotz resonator, the control system's electrical resonance is tuned to the cavity resonance (Mao and Pietrzko [2005]; Pietrzko and Mao [2008]). This approach uses a combination of cavity and panel control. The possibility of inserting actuators and sensors into structures has led to the development of Active Structural Acoustic Control (ASAC). Elliott (Elliott and Johnson [1993]) showed that at low frequencies, sound power is produced mainly by the first radiation mode (piston-motion). Reducing in the volume velocity of the panel leads to a reduction in sound power. Sensor networks (Sors and Elliott [2002]; Francois et al. [2001] ) or a distributed sensor such as PVDF film (Preumont et al. [2005]) have been implemented to evaluate volume velocity and supply a SISO controller. System stability is guaranteed (Preumont [2002]) when the actuators and the sensors are ideal, collocated and dual. Model-based strategies have also been introduced in vibroacoustic control. Baumann [1991]; Baumann et al. [1992] proposed to compute sound power using radiation filters introduced into the state space model of the structure, with the acoustic energy being incorporated into the cost function. Experiments carried out by Bingham et al. [2001] and Dehandschutter et al. [1999] have demonstrated the validity of this method, although it is limited by the number of radiation filters which need to be introduced into the state space formulation. Below the critical frequency, transmission is mass controlled (Fahy [1985]). Alujevic and Gardonio [2006] showed that a light panel, controlled by an active virtual mass with decentralized, collocated MIMO controllers, behaves like a heavy, non-controlled panel. Although the transmission losses are increased, sound power peaks persist due to the lack of active damping.

Contrary to simple panels, double panels present very good acoustic properties at medium to high frequencies, due to coupling effects between the plates and the cavity. Unfortunately, double panels are not very effective at low frequencies. The main advantage of combining a double panel with active control is that good acoustic properties can be obtained over the whole frequency band. Moreover, the double panel has the advantage of filtering out the eventual gain peaking or spillover. Spillover appears at high frequency in the case of a modal control approach due to the use of a reduced model of the structure. For all of these reasons, the combination of a double panel with active control appears to be a highly attractive solution.

The aim of this paper is to describe the reduction in the transmission of sound through a double panel by modifying its modal distribution. When structures are rela- 
tively small and light, the associated panels have a low modal overlap. Modal control thus appears to be well adapted to this situation, since it allows the active surface and the number of control components to be limited (Gaudiller and Der Hagopian [1996]) and the control energy to be concentrated on high radiation efficiency modes. Moreover, the use of nonlinear algorithms allows the control energy to be reduced, through the use of a variable gain, which is adjusted according to the level of modal quantities in order to improve the energy exchange (Gaudiller and Matichard [2007]) or by removing the restoration of potential ( Matichard and Gaudiller [2005]). By adding modal masses, the resonance frequencies are individually shifted to a less audible frequency range. Also, the mass control of one eigenmode can be as achieved through the addition of a virtual modal mass. The acoustic characteristics of the structure should also be improved in the upper frequency range, thereby increasing its transmission losses. This paper presents a modal state space feedback controller which acts on modal mass and modal damping. Section 2 describes the modeling of the structure and the method used to compute the sound power. Section 3 introduces the vibroacoustic controller driven by modal accelerations and modal velocities. Simulations of this "Mass and Damping vibroacoustic modal control" is presented in the case of a double panel in section 4. It is then compared with other vibroacoustic modal control techniques.

\section{Modeling}

The first step in the design of a model-based control strategy consists in building a structural and acoustic model. This model is used by the observer to reconstruct the modal state vector of the structure from sensor signals. The control gains are then applied to the estimated state vector, and can be optimized by minimizing the system's kinetic energy and radiated sound power.

\subsection{Double panel description}

In the low frequency domain, the natural wavelength is much greater than the distance between the two plates, such that coupling due to the air space can be approximated by a uniformly distributed air spring. The governing equations for two simply supported plates coupled through a linear spring can be written as (Vaicaitis [1983]) :

$$
\begin{aligned}
m_{I} \ddot{w}_{I}+C_{I} \dot{w}_{I}+D_{I} \nabla^{4} w_{I}+K_{s}\left(w_{I}-w_{R}\right)+\frac{1}{3} m_{s} \ddot{w}_{I}+\frac{1}{6} m_{s} \ddot{w}_{R} & =p_{I}(x, y, t),(1) \\
m_{R} \ddot{w}_{R}+C_{R} \dot{w}_{R}+D_{R} \nabla^{4} w_{R}+K_{s}\left(w_{R}-w_{I}\right)+\frac{1}{3} m_{s} \ddot{w}_{R}+\frac{1}{6} m_{s} \ddot{w}_{I} & =p_{R}(x, y, t),(2)
\end{aligned}
$$

with $w_{I}$ and $w_{R}$ being respectively the transverse displacement of the incident (subscript $I$ ) and radiating $(R)$ plates, $\nabla^{4}$ the bi-Laplacian operator, $K_{s}$ the air-layer stiffness, $p_{I}(x, y, t)$ and $p_{R}(x, y, t)$ the normal external pressure acting on the incident and radiating plates and $C_{I}$ and $C_{R}$ the structural damping coefficients of the plates. Subscript $s$ denotes the air cavity, $\frac{m_{s}}{3}$ and $\frac{m_{s}}{6}$ are the contributions of the air cavity mass apportioned to each of the two plates. The mass density per unit area of the structure and the air stiffness per unit area are defined as follows :

$$
\begin{gathered}
m_{I}=\rho_{I} \cdot h_{I} ; m_{R}=\rho_{R} \cdot h_{R} ; m_{s}=\rho_{s} \cdot h_{s}, \\
D_{I}=\frac{E_{I} h_{I}^{3}}{12\left(1-v_{I}^{2}\right)} ; D_{R}=\frac{E_{R} h_{R}^{3}}{12\left(1-v_{R}^{2}\right)}, \\
K_{s}=\frac{\rho_{s} c^{2}}{h_{s}} ; \nabla^{4}=\frac{\partial^{4}}{\partial x^{4}}+2 \frac{\partial^{4}}{\partial x^{2} \partial y^{2}}+\frac{\partial^{4}}{\partial y^{4}},
\end{gathered}
$$

where $E$ is Young's modulus, $v$ is the Poisson's ratio, $\rho$ is the mass density and $h$ is the thickness of the plate. 
For the sake of simplicity, the following new terms are introduced :

$$
a_{I}=m_{I}+\frac{m_{s}}{3} ; a_{R}=m_{R}+\frac{m_{s}}{3} ; b_{s}=\frac{m_{s}}{6} .
$$

When the structure is lightly damped and the modes are sufficiently decoupled, the transverse displacement fields can written in a matrix form. The linear system can then be described by a set of decoupled modal equations after a change of variable :

$$
w=\phi q
$$

with $w$ the transverse displacement matrix, $\phi$ the modal shapes and $q$ the modal displacement amplitudes. In the case of two simply supported plates (dimensions : $L_{x}$, $L_{y}$ ), the dynamic behavior of both plates are similar and can be written using a modal approach where the eigen-shapes are given by :

$$
\phi_{m n}^{I}(x, y)=\phi_{m n}^{R}(x, y)=\phi_{m n}(x, y)=\sin \left(\frac{m x \pi}{L_{x}}\right) \sin \left(\frac{n y \pi}{L_{y}}\right) .
$$

The displacements of the incident and radiating plates can be written in the form of a linear combination of modes :

$$
w_{I}=\sum_{m=0}^{m=\infty} \sum_{n=0}^{n=\infty} q_{m n}^{I} \phi_{m n}^{I}
$$

and

$$
w_{R}=\sum_{m=0}^{m=\infty} \sum_{n=0}^{n=\infty} q_{m n}^{R} \phi_{m n}^{R} .
$$

From (1), (2) and (7), for the two plates, the modal equations of motion of mode $m n$ are given by :

$$
\begin{aligned}
& \ddot{q}_{m n}^{I}+2 \xi_{m n} \omega_{m n}^{I} \frac{m_{I}}{a_{I}} \dot{q}_{m n}^{I}+\left(\left(\omega_{m n}^{I}\right)^{2} \frac{m_{I}}{a_{I}}+\frac{K_{s}}{a_{I}}\right) q_{m n}^{I}-\frac{K_{s}}{a_{I}} q_{m n}^{R}+\frac{b_{s}}{a_{I}} \ddot{q}_{m n}^{R}=\frac{P d_{m n}^{I}}{a_{I}} \\
& \ddot{q}_{m n}^{R}+2 \xi_{m n} \omega_{m n}^{R} \frac{m_{R}}{a_{R}} \dot{q}_{m n}^{R}+\left(\left(\omega_{m n}^{R}\right)^{2} \frac{m_{B}}{a_{R}}+\frac{K_{s}}{a_{R}}\right) q_{m n}^{R}-\frac{K_{s}}{a_{R}} q_{m n}^{I}+\frac{b_{s}}{a_{R}} \ddot{q}_{m n}^{I}=\frac{P d_{m n}^{R}}{a_{R}}
\end{aligned}
$$

where $\xi_{m n}$ is the modal damping factor, and $\omega_{R}^{m n}$ and $\omega_{I}^{m n}$ are the eigen-frequencies of the uncoupled plates :

$$
\omega_{m n}^{R}=\sqrt{\frac{D_{R}}{m_{R}}}\left[\left(\frac{m \pi}{L_{x}}\right)^{2}+\left(\frac{n \pi}{L_{y}}\right)^{2}\right], \omega_{m n}^{I}=\sqrt{\frac{D_{I}}{m_{I}}}\left[\left(\frac{m \pi}{L_{x}}\right)^{2}+\left(\frac{n \pi}{L_{y}}\right)^{2}\right],
$$

and $P d_{m n}^{I}, P d_{m n}^{R}$ the generalized external forces :

$$
\begin{gathered}
P d_{m n}^{I}=\frac{4}{L_{x} \cdot L_{y}} \int_{0}^{L_{x}} \int_{0}^{L_{y}} \phi_{m n}^{I}(x, y) p_{I}(x, y, t) d x d y \\
P d_{m n}^{R}=\frac{4}{L_{x} \cdot L_{y}} \int_{0}^{L_{x}} \int_{0}^{L_{y}} \phi_{m n}^{T}(x, y) p_{R}(x, y, t) d x d y .
\end{gathered}
$$

\section{State space matrices of both plates}

The Fourier transform of the coupling terms between the two plates in (11) and (12) can be simplified when $\omega<<\omega_{c}$ with $\omega_{c}<\sqrt{\frac{K_{s}}{b_{s}}}$ by :

$$
\begin{aligned}
& \frac{-\hat{q}_{m n}^{R}}{a_{I}}\left(K_{s}+\omega^{2} b_{s}\right) \approx \frac{-K_{s}}{a_{I}} \hat{q}_{m n}^{R}, \\
& \frac{-\hat{q}_{m n}^{I}}{a_{R}}\left(K_{s}+\omega^{2} b_{s}\right) \approx \frac{-K_{s}}{a_{R}} \hat{q}_{m n}^{I},
\end{aligned}
$$


where $\hat{q}_{m n}^{I}$ and $\hat{q}_{m n}^{R}$ denote the Fourier transform of the amplitudes of the incident and radiating plates for the mode $m n$. These simplifications result from the fact that the inertia of the air-layer is negligible when compared to the influence of its stiffness.

In this study, frequencies higher than $\omega_{c}$ are not considered. From (11), (12), (15), (16), the state space matrices of the plates coupled by an air cavity can be written as :

$$
\begin{aligned}
& \underbrace{\left[\begin{array}{c}
q_{m n}^{\dot{I}} \\
q_{m}^{\dot{R}} \\
q_{m n}^{\dot{I}} \\
q_{m n}^{\dot{R}}
\end{array}\right]}_{\dot{X}}=\underbrace{\left[\begin{array}{cccc}
0 & 0 & 1 & 0 \\
0 & 0 & 0 & 1 \\
-\frac{K_{s}+m_{I}\left(\omega_{m n}^{I}\right)^{2}}{a_{I}} & \frac{K_{s}}{a_{I}} & -2 \xi_{m n}^{I} \omega_{m n}^{I} \frac{m_{I}}{a_{I}} & 0 \\
\frac{K_{s}}{a_{R}} & -\frac{K_{s}+m_{R}\left(\omega_{m n}^{R}\right)^{2}}{a_{R}} & 0 & -2 \xi_{m n}^{R} \omega_{m n}^{R} \frac{m_{R}}{a_{R}}
\end{array}\right]}_{A} \cdot \underbrace{\left[\begin{array}{c}
q_{m n}^{I} \\
q_{m n}^{R} \\
q_{m n}^{\dot{I}} \\
q_{m n}^{\dot{R}}
\end{array}\right]}_{X} \\
& +\underbrace{\left[\begin{array}{c}
0 \\
0 \\
\frac{P d_{m n}^{I}}{a_{I}} \\
0
\end{array}\right]}_{E_{I}} W_{I}+\underbrace{\left[\begin{array}{c}
0 \\
0 \\
0 \\
\frac{P d_{m n}^{R}}{a_{R}}
\end{array}\right]}_{E_{R}} W_{R}+\underbrace{\left[\begin{array}{c}
0 \\
0 \\
\frac{B_{m n}^{I}}{a_{I}} \\
0
\end{array}\right]}_{B_{I}} U_{I}+\underbrace{\left[\begin{array}{c}
0 \\
0 \\
0 \\
\frac{B_{m n}^{R}}{a_{R}}
\end{array}\right]}_{B_{R}} U_{R}
\end{aligned}
$$

where $X$ is the modal state vector, $A$ is the dynamic matrix, $E_{I}$ and $E_{R}$ are the disturbance state matrices, $B_{I}$ and $B_{R}$ are the actuation state matrices, $W_{I}$ and $W_{R}$ represent the system noise, and $U_{I}$ and $U_{R}$ represent the control vectors.

\section{State space matrices of the global structure}

When the double panel is excited by an acoustic wave on the incident plate, the modal displacements of each plate are obtained in state space formulation as :

$$
y=C x,
$$

where $\mathrm{C}$ is the output matrix which depends on the sensors. In the Laplace domain, if incident pressure only is considered, the outputs are computed as :

$$
Y(s)=C(s I-A)^{-1} E_{I} W_{I}(s),
$$

For each mode, transfer functions from excitation (aerial or mechanical) to the outputs of each plate can be obtained from the summation of the two second order rational fraction polynomials (20) and (21) indexed 1 and 2. Indeed, each modal shape $m n$ has two resonances, corresponding to these two fraction polynomials (with both plates in-phase, or out-of-phase). These fractions can be computed analytically or obtained experimentally by curve fitting identification (Richardson and Formanti [November 1982.]) :

$$
\begin{aligned}
q_{m n}^{I}(s) & =\frac{\psi_{1}^{I} e^{j \phi_{1}^{I}}}{s^{2}+b_{I} s+c_{I}}+\frac{\psi_{2}^{I} e^{j \phi_{2}^{I}}}{s^{2}+e_{I} s+f_{I}}, \\
q_{m n}^{R}(s) & =\frac{\psi_{1}^{R} e^{j \phi_{1}^{R}}}{s^{2}+b_{R} s+c_{R}}+\frac{\psi_{2}^{R} e^{j \phi_{2}^{R}}}{s^{2}+e_{R} s+f_{R}} .
\end{aligned}
$$

where $\psi$ 's are the amplitudes, c's and f's are the eigen-frequencies, b's and e's are the terms relative to damping and $\phi$ 's are the phases. In the case of a perfect double panel, $f=f_{I}=f_{R}, c=c_{I}=c_{R}, \phi_{1}^{I}=\phi_{2}^{I}=0$ and $\phi_{1}^{R}=\phi_{2}^{R}-\pi=0$. The relative modal amplitudes of both plates at each resonance frequency are given by $\psi_{1}^{I}, \psi_{1}^{R}, \psi_{2}^{I}$ and $\psi_{2}^{R}$. Since both plates have the same modal damping ( $b$ at the in-phase frequencies, and $e$ at the out-of-phase frequencies) and the modal quantities $q$ are independent, the global state can be written as :

$$
\left[\begin{array}{c}
s . q_{m n}^{1 \text { global }} \\
s . q_{m n}^{2 g l o b a l} \\
s^{2} . q_{m n}^{1 g l o b a l} \\
s^{2} . q_{m n}^{2 g l o b a l}
\end{array}\right]=\left[\begin{array}{cccc}
0 & 0 & 1 & 0 \\
0 & 0 & 0 & 1 \\
-c & 0 & -b & 0 \\
0 & -f & 0 & -e
\end{array}\right] \cdot\left[\begin{array}{c}
q_{m n}^{1 g l o b a l} \\
2 g l o b a l \\
q_{m n}^{19 l o b a l} \\
s . q_{m n}^{2 g l a b a l} \\
s . q_{m n}^{2 g l o b a l}
\end{array}\right]+\left[\begin{array}{l}
0 \\
0 \\
1 \\
1
\end{array}\right] U_{I}(s) .
$$




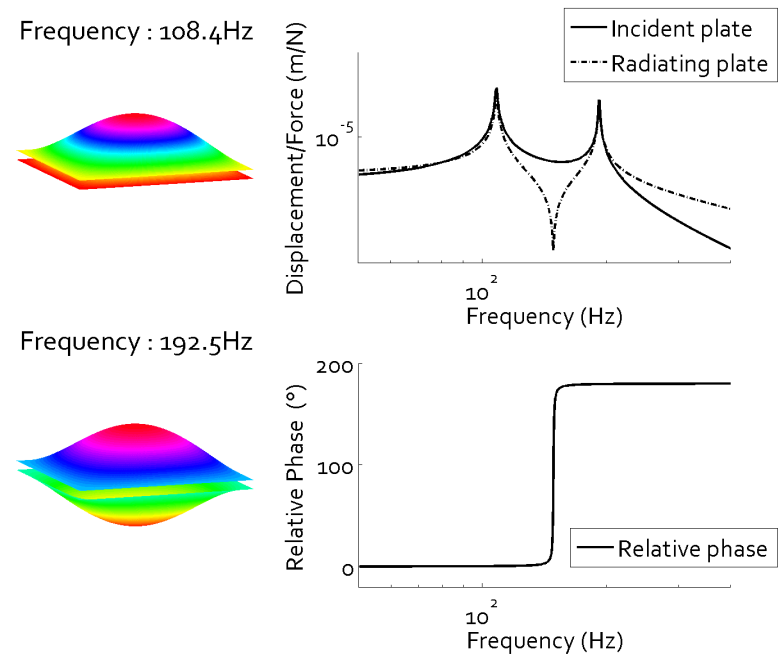

Figure 1 - Modal displacements of the incident plate $q_{I}^{1,1}$ and of the radiating plate $q_{R}^{1,1}$, and their relative phase

The relationship between the modal displacements of the separated plates and those of the global structure is given by :

$$
\begin{gathered}
q_{m n}^{I}(s)=\psi_{1}^{I} \cdot q_{m n}^{1 \text { global }}(s)+\psi_{2}^{I} \cdot q_{m n}^{2 g l o b a l}(s), \\
q_{m n}^{R}(s)=\psi_{1}^{R} \cdot q_{m n}^{1 \text { global }}(s)-\psi_{2}^{R} \cdot q_{m n}^{2 g l o b a l}(s) .
\end{gathered}
$$

In equation (24), the negative sign preceding the last term indicates that two panels are out of phase at the second resonance $\left(\phi_{2}^{R}=\pi\right)$. The modal displacements of the incident plate $q_{1,1}^{I}$ and of the radiating plate $q_{1,1}^{R}$ are plotted in figure 1 . The same modal shape appears twice; once when the two plates are out of phase, once when they are in-phase; with different relative amplitudes.

\subsection{Actuators}

The control actuators considered in this study are square PZT ceramics bonded to the structure. The generalized forces exerted by these actuators introduced into the actuation state matrices $B_{I}$ and $B_{R}$, are given for the mode $m n$ and the patch $p$ by (Dimitriadis and Fuller [1991]) :

$$
B_{m n}^{p}=4 C_{0} \epsilon_{p}\left(-\frac{\alpha_{x}^{2}+\alpha_{y}^{2}}{\alpha_{x} \alpha_{y}}\right)\left(\cos \left(\alpha_{x} x_{1}\right)-\cos \left(\alpha_{x} x_{2}\right)\right)\left(\cos \left(\alpha_{y} y_{1}\right)-\cos \left(\alpha_{y} y_{2}\right)\right)
$$

with

$$
\begin{array}{r}
\alpha_{x}=\frac{m \pi}{L_{x}} ; \alpha_{y}=\frac{n \pi}{L_{y}} ; \epsilon_{p}=\frac{d_{31} u}{t_{a}}, \\
C_{0}=-E \frac{1+v_{a}}{1-v} \frac{P}{1+v-\left(1+v_{a}\right) P} \frac{2}{3}\left(\frac{h}{2}\right)^{2}, \\
P=-\frac{E_{a}}{E} \frac{1-v^{2}}{1-v_{a}^{2}} \frac{3 t_{a}(h / 2)\left(h+t_{a}\right)}{2\left[(h / 2)^{3}+t_{a}^{3}\right]+3(h / 2) t_{a}^{2}},
\end{array}
$$

where $B_{m n}^{p}$ is the generalized force of the $p^{t h}$ actuator at mode m-n, $\mathrm{u}$ is the control voltage, $d_{31}$ is the piezoelectric transverse coefficient, $C_{0}$ is the capacitance, $\left[x_{1}, x_{2}\right],\left[y_{1}, y_{2}\right]$ are the coordinates of the square actuator, $v, v_{a}$ are respectively the Poisson's ratio of the plate and the actuator, $h$ and $t_{a}$ are the plate and actuator thicknesses, and $E$ and $E_{a}$ are their Young moduli. 


\subsection{Acoustic model}

An acoustic model is required in order to optimize the control gains of the vibroacoustic controller and to evaluate the performances of the control system.

\subsubsection{Excitation by a diffuse field}

Let us consider the excitation to be an incident acoustic plane wave. The expression of the acoustic pressure due to the plane wave on the surface is (Roussos [1985]) :

$$
p_{i}(x, y, t)=P_{i} e^{j\left(\omega t-k x \sin \theta_{i} \cos \phi_{i}-k y \sin \theta_{i} \sin \phi_{i}\right)},
$$

where $P_{i}$ is the incident pressure amplitude, $\theta_{i}$ and $\phi_{i}$ are the incidence angles, $k$ is the acoustic wavelength and $\omega$ is the frequency. Since the coupling is weak between the fluid at the incident surface (air) and the incident plate, the excitation can be considered to be the blocked pressure, corresponding to twice the incident pressure :

$$
p_{B}(x, y)=2 p_{i}(x, y)=\sum_{m=1}^{M} \sum_{n=1}^{N} P d_{m n} \sin \left(\frac{m \pi x}{l_{x}}\right) \sin \left(\frac{n \pi y}{l_{y}}\right),
$$

where $P d_{m n}$ are the modal amplitudes defined by :

$P d_{m n}\left(\theta_{i}, \phi_{i}\right)=\int_{0}^{l_{x}} \int_{0}^{l_{y}} \frac{8}{L_{x} \cdot L_{y}} P_{i} e^{j \sin \theta_{i}\left(-k x \cos \phi_{i}-k y \sin \phi_{i}\right)} \sin \left(\frac{m \pi x}{l_{x}}\right) \sin \left(\frac{n \pi y}{l_{y}}\right) d(x 2 y)$.

The development of $P d_{m n}\left(\theta_{i}, \phi_{i}\right)$ is provided by Roussos [1985]. The incident intensity is written :

$$
I_{i}=\frac{P_{i}^{2} \cos \phi_{i}}{2 \rho c} .
$$

and the incident acoustic power can be computed from the intensity as :

$$
W_{i}=\int I_{i} \cdot d S
$$

In the case of a diffuse field, the generalized forcing pressure $P d_{m n}^{\text {diffuse }}$ can be computed without weighting coefficients, as :

$$
P d_{m n}^{\text {diffuse }}=\int_{-\frac{\pi}{2}}^{\frac{\pi}{2}} \int_{0}^{\pi} P d_{m n}\left(\theta_{i}, \phi_{i}\right) d \theta d \phi .
$$

This approximation is sufficiently accurate for the purposes of active control simulations.

\subsubsection{Radiated pressure}

The acoustic power $W_{r}$ can be computed using the well-known Rayleigh integral or the radiation resistance matrix of the structural modes $M$ and the modal velocities (Elliott and Johnson [1993]). This formulation allows the radiation to be introduced into the state space formulation of the double panel :

$$
W_{r}=\dot{q}^{H} M \dot{q},
$$

where the superscript ${ }^{H}$ denotes the Hermitian. $M$ can be calculated from the radiation resistance matrix of elemental radiators and modal shapes. The structure is discretized into elementary radiators radiating into free space, which are small compared to the acoustic wave length. The sound power radiated by a set of elemental sources is calculated as :

$$
W_{r}=\frac{S_{e}}{2} \Re\left\{v^{H} p\right\}=v^{H} R v,
$$


with

$$
R_{i j}=\frac{S_{e}}{2} \Re\left\{Z_{i j}\right\}=\frac{\omega^{2} \rho S_{e}^{2}}{4 \pi c} \frac{\sin k r_{i j}}{k r_{i j}}
$$

where $v$ and $p$ are respectively the velocity and pressure vectors of the radiators, $k$ is the acoustic wave length, $S_{e}$ is the surface area of the radiator, c is the speed of sound in the medium, $\omega$ is the frequency, $\rho$ is the mass density of air and $r_{i j}$ denotes the distance between elemental sources. $\Re$ indicates the real part and $Z$ and $R$ are respectively the acoustic transfer impedance and the radiation resistance matrices of the elemental radiators. After the change of variable defined in equation(7), $M$ and $W_{r}$ become:

$$
\begin{aligned}
W_{r}(\omega) & =v^{H} R(\omega) v=\dot{q}^{H} \phi^{H} R(\omega) \phi \dot{q}, \\
M(\omega) & =\phi^{H} R(\omega) \phi .
\end{aligned}
$$

Then, for any structures and any set of frequencies, the radiation resistance matrix of the structural modes $M$ can be calculated and approximated using a Laplace-domain multiple-input, multiple output transfer function $M(s) . M(s)$ is then factorized into a stable causal radiation filter $G(s)(40)$, and $W(s)$ is given by (41) :

$$
\begin{aligned}
M(s) & =G^{T}(-s) G(s), \\
W_{r}(s) & =\dot{Q}_{r}(-s)^{T} M(s) \dot{Q}_{r}(s),
\end{aligned}
$$

where $\dot{Q}_{r}(s)$ are the modal velocities of the radiating plate in the Laplace domain.

\section{Transmission Loss (TL)}

In the following, the acoustic transmission loss factor TL is computed according its definition :

$$
T L=\log \frac{W_{i}}{W_{r}} .
$$

\subsubsection{Introduction of acoustics into the state-space formulation}

The radiation filters $(\mathrm{RF}) G(s)$ are converted into a state space form and then introduced into an augmented state space of the system (Baumann [1991]; Baumann et al. [1992]) :

$$
\left\{\begin{array}{l}
\dot{r}=A_{G} r+B_{G} \dot{q} \\
z=C_{G} r+D_{G} \dot{q}
\end{array},\right.
$$

with $z$ being the result of the velocity components passing through the radiation filter. $z^{2}$ is the sound power.

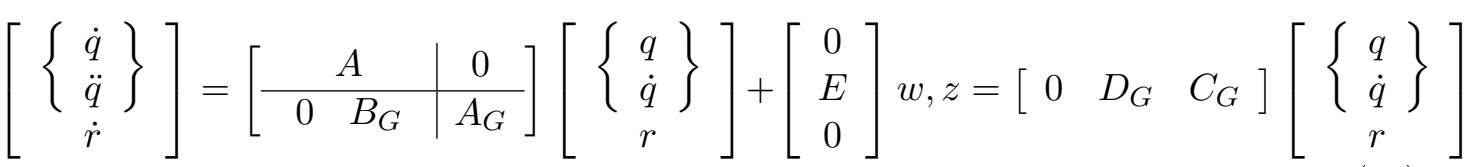




\section{Control}

In the framework of small and lightly damped structures, modal control can be effective due to low modal overlap. When the panel is excited by an acoustic wave, the excitation level is frequency dependent and varies as a function of the mode indices. The low order modes (odd - odd) are highly excited. Likewise, these modes are responsible for most of the radiated sound power, such that by controlling only these highly excited and highly radiating modes, a substantial reduction in sound power can be achieved. Modal control allows focussing control energy exclusively on these modes, using a small number of actuators, while requiring a small number of sensors for modal reconstruction (Gaudiller and Der Hagopian [1996]).

When a structure is excited by a diffuse field below its critical frequency, the transmission is "mass controlled" (Fahy [1985]). The mass addition effect at low frequencies lowers the resonance frequencies to a less audible frequency range, while reducing vibration amplitudes, and the overall transmission loss is increased. In the case of finite structures with modal behavior, transmission losses, below the critical frequency, are also governed by damping. Consequently, actively controlling mass and damping modifies the modal distribution of the panel. The natural frequencies of the high radiation modes can be lowered through the addition of an active modal mass. At resonance frequencies, the amplitude of the vibrations can be reduced by modal damping. .

The double panel, equipped with actuators and sensors is excited by a diffuse field (Figure 2). The modal control strategy used is illustrated in Figure 3. The controller is driven by modal accelerations and modal velocities. Sensor signals from the structure (a) are used for modal reconstruction using a modal observer (d) following which the modal state (displacement and velocities) is derived (c). Then, the modal control gains (b) are applied in to the "observed" modal velocities and accelerations to generate active modal damping and active modal mass.
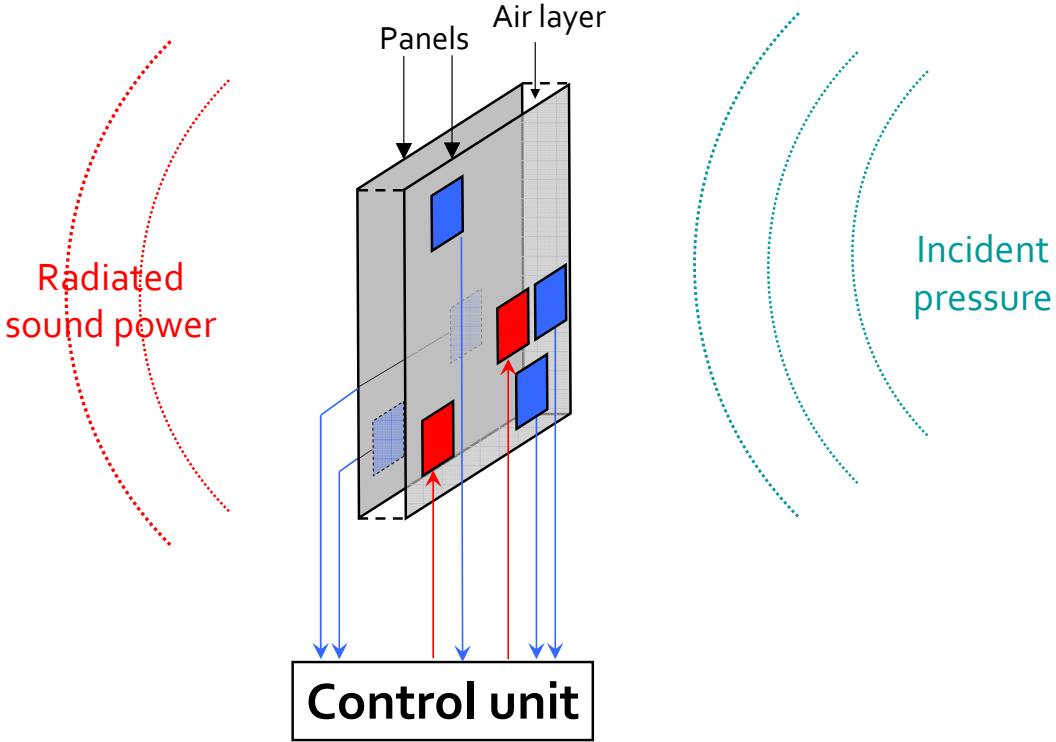

FiguRE 2 - Design of the control system for the double panels. Red squares : actuators, Blue squares : sensors. 
(a)

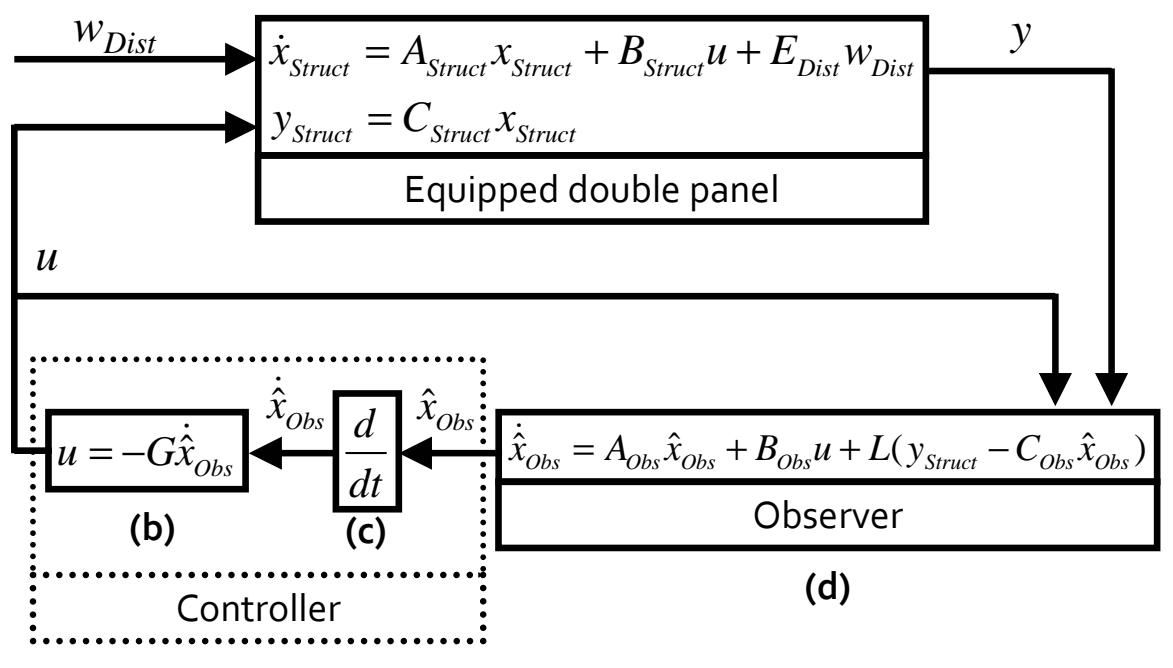

Figure 3 - Modal Control scheme

\subsection{Controller}

The control is driven by modal accelerations and modal velocities obtained from the derivation of the reconstructed state vector. The active modal mass can be considered as a virtual mass concentrated on controlled modes. Consequently, the amplitudes of mass controlled modes are reduced beyond resonances due to frequency shifts. The control $u$ is given by :

$$
u=-K \dot{x}_{c}=-\sum_{k=1}^{N_{c}}\left[K_{v}(k) \dot{q}_{k}+K_{a}(k) \ddot{q}_{k}\right],
$$

with $x_{c}$ the state vector of the controlled modes, $K_{a}(k)$ and $K_{v}(k)$ the matrix gains relative to modal accelerations and velocities at the $k^{\text {th }}$ mode. Then, the active mass and damping can be expressed in the modal equation as :

$$
\begin{gathered}
{[1+\underbrace{K_{a}(k) \cdot B_{k}^{p}}_{\text {Active Modal Mass }}] \ddot{q}_{k}+[2 \xi_{k} \omega_{k}+\underbrace{K_{v}(k) \cdot B_{k}^{p}}_{\text {Active Modal Damping }}] \dot{q}_{k}+\omega_{k}^{2} q_{k}=} \\
E_{k} W_{D i s t}-\underbrace{B_{k}^{p} \sum_{k \neq l} K_{v}(l) \dot{q}_{l}+K_{a}(l) \ddot{q}_{l}}_{\text {Excitation of controlled modes }} .
\end{gathered}
$$

The reconstructed state feeds the controller, whose control gain matrix $K$ is computed with an optimal control algorithm, using an energetic cost function which takes the control energy and vibration (47) or sound power (48), into consideration. These are defined as :

$$
\begin{gathered}
J=\int_{0}^{\infty}\left(x^{T} Q x+u^{T} R u\right) d t \\
J_{R a d}=\int_{0}^{\infty}\left(z^{T} z+u^{T} R u\right) d t \text { with } z=\mathcal{L}^{-1}\left(G(s) \dot{Q}_{r}(s)\right),
\end{gathered}
$$

where $\mathrm{Q}$ and $\mathrm{R}$ are the weighting matrices. Note that in the case of sound power control, some non-radiating modes may be highly excited. 


\subsection{Modal reconstruction}

Several methods may be used for modal control. Here, in order to minimize the number of sensors, a Luenberger observer (Luenberger [1966]) is preferred to modal filter techniques requiring a large observation area, and as many sensors as modes need to be observed in order to achieve accurate modal reconstruction. The observer estimates the state variables in real time (Figure $3(\mathrm{~d})$ ). It uses a reduced model of the structure and a proportional control (Matrix gain L) that allows the error between the actual and estimated sensor outputs to be minimized. The observer is tuned such that its dynamics are between two and five times faster than those of the controller, in order to minimize noise amplification effects. The reconstructed state $\hat{x}_{O b s}$ is estimated from :

$$
\dot{\hat{x}}_{\text {Obs }}=A_{\text {Obs }} \hat{x}_{\text {Obs }}+B_{\text {Obs }} u+L\left(y_{\text {Struct }}-C_{\text {Obs }} \hat{x}_{\text {Obs }}\right),
$$

in which $y_{\text {Struct }}$ describes the sensor outputs given by the "non reduced" model :

$$
\begin{aligned}
& \dot{\hat{x}}_{\text {Struct }}=A_{\text {Struct }} \hat{x}_{\text {Struct }}+B_{\text {Struct }} u+E_{\text {Struct }} w_{\text {Struct }} \\
& y_{\text {Struct }}=C x_{\text {Struct }},
\end{aligned}
$$

where $A_{\text {Struct }}$ is the "structure state matrix" and $A_{O b s}$ is the "observer state matrix" used for the modal reconstruction. $B_{\text {Struct }}, B_{O b s}, C_{S t r u c t}$ and $C_{O b s}$ are respectively the structure and observer actuation state matrices $(B)$ and observation matrices $(C)$. $\mathrm{G}$ and $\mathrm{L}$ are respectively the control gain and observer gain matrices. In practice, the number of modes reconstructed through the observer is limited. In simulations, this number is usually smaller than the number of modes considered in the state space system. These numbers depend directly on the control objectives and the number of sensors.

\subsection{Observed controlled structure}

The state of the controlled structure can be written as :

$X_{\text {Struct }}(s)=\left[s\left[I+B_{\text {Struct }} K\left[s\left[I+B_{\text {Obs }} K\right]-A_{\text {Obs }}+L C_{\text {Obs }}\right]^{-1} L C_{\text {Struct }}\right]-A_{\text {Struct }}\right]^{-1} E_{\text {Struct }} W_{\text {Struct }}(s)$.

During steady state, the control signal is given by :

$$
U(s)=-K\left[s\left(I+B_{O b s} K\right)-A_{O b s}+L C_{O b s}\right]^{-1} L C_{\text {Struct }} s X_{\text {Struct }}(s) .
$$




\section{Application}

\subsection{The model}

In this simulation, 25 modes are used to describe the double panel. Sensor patches are located on both plates ( 3 on the incident plate and 2 on the radiating plate). Their locations allow a good modal coupling coefficients for observed modes. The modal observer allows reconstructing the state of the first eleven modes ( 0 to $500 \mathrm{~Hz})$ from the five sensors. Then, the 2 actuators bonded to the incident plate are used to control the first five modes (10 resonances) within the $[0 ; 350 \mathrm{~Hz}]$ frequency band. Their locations were chosen to maximize the coupling coefficients with the radiating modes. Moreover, by positioning actuators on the incident plate, it is possible to take advantage of the passive properties of the double panel in the case of eventual spillover. The double panel and PZT properties used in the simulation are provided in table 1.

\begin{tabular}{|l|l||l|l|}
\hline \hline Plate density & $\rho_{\text {Plates }}=2700 \mathrm{Kg} / \mathrm{m}^{3}$ & Plate width & $L_{x}=0.3 \mathrm{~m}$ \\
Plate Young's modulus & $E=69 * 10^{9} \mathrm{~Pa}$ & Plate length & $L_{y}=0.38 \mathrm{~m}$ \\
Modal damping ratio & $\xi_{m n}=0.005$ & Top Plate thickness & $h_{I}=0.001 \mathrm{~m}$ \\
Air density & $\rho_{s}=1.23 \mathrm{Kg} / \mathrm{m}^{3}$ & Bottom Plate thickness & $h_{R}=0.0016 \mathrm{~m}$ \\
Plate Poisson's ratio & $v_{B}=v_{T}=0.33$ & Air cavity thickness & $h_{s}=0.084 m$ \\
\hline Patch density & $\rho_{\text {patch }}=7600 \mathrm{Kg} / \mathrm{m}^{3}$ & Patch width & $l=0.07 m$ \\
Patch Poisson's ratio & $v_{\text {patch }}=0.3$ & Patch length & $L=0.07 m$ \\
Patch Young's modulus & $E_{a}=60 * 10^{9} \mathrm{~Pa}$ & Patch thickness & $t_{a}=0.001 \mathrm{~m}$ \\
\hline Position Actuator 1 & $X_{A 1}=0.04 ; Y_{A 1}=0.04$ & Position Actuator 2 & $X_{A 2}=0.155 ; Y_{A 2}=0.115$ \\
(m) & $Z_{A 1}=0$ & (m) & $Z_{A 2}=0$ \\
\hline Position Sensor 1 & $X_{S 1}=0.04 ; Y_{S 1}=0.04$ & Position Sensor 2 & $X_{S 2}=0.155 ; Y_{S 2}=0.115$ \\
(m) & $Z_{S 1}=8.4 e^{-3}$ & (m) & $Z_{S 2}=8.4 e^{-3}$ \\
\hline Position Sensor 3 & $X_{S 3}=0.3 ; Y_{S 3}=0.08$ & Position Sensor 4 & $X_{S 4}=0.16 ; Y_{S 4}=0.24$ \\
(m) & $Z_{S 3}=0$ & (m) & $Z_{S 4}=0$ \\
\hline Position Sensor 5 & $X_{S 5}=0.04 ; Y_{S 5}=0.2$ & Disturbance level & $96 \mathrm{~dB}$ \\
$(\mathrm{~m})$ & $Z_{S 5}=0$ & & \\
\hline \hline
\end{tabular}

TABLE 1 - Simulation parameters

In the case of a diffuse field disturbance in the low frequency domain, odd-odd modes are highly excited and contribute significantly to the radiated sound power due to their high radiation efficiency. Consequently, controlling these modes may lead to an considerable reduction of transmitted sound. The radiation resistance matrix of the structural modes of a double panel is presented in figure 4 . This figure shows that levels and slopes of the self radiation (diagonal terms) and mutual radiation (non-diagonal terms) differ depending on the mode indices.

\subsection{Results}

Usually, for simple structures, the transmission losses of the Frequency Weighted (FW) and Radiation Filter (RF) controllers have the same characteristics Lhuillier et al. [2008]. They provide good isolation at resonance frequencies. Consequently, in figure 5, presenting the transmission loss index, the downwards peaks disappear, but the general behavior of the TL does not change.

In these simulations, the mass - damping control strategy uses damping gains stemming from (48) and an active mass is added to the first mode (1-1) due to its strong contribution to radiated sound power at low frequencies.

In figure 5, the transmission losses of the uncontrolled and controlled double panel are shown (for Mass-damping and vibro-acoustic control). The modelled, controlled and observed modes are indicated by crosses, diamonds and circles, respectively. The strong increase of the transmission loss index at the first resonance frequencies is due to an increase in modal damping (from $0.5 \%$ to $6 \%$ for the first mode). The influence of an additional mass can be seen to result in a lowering of its frequency, mainly in the 


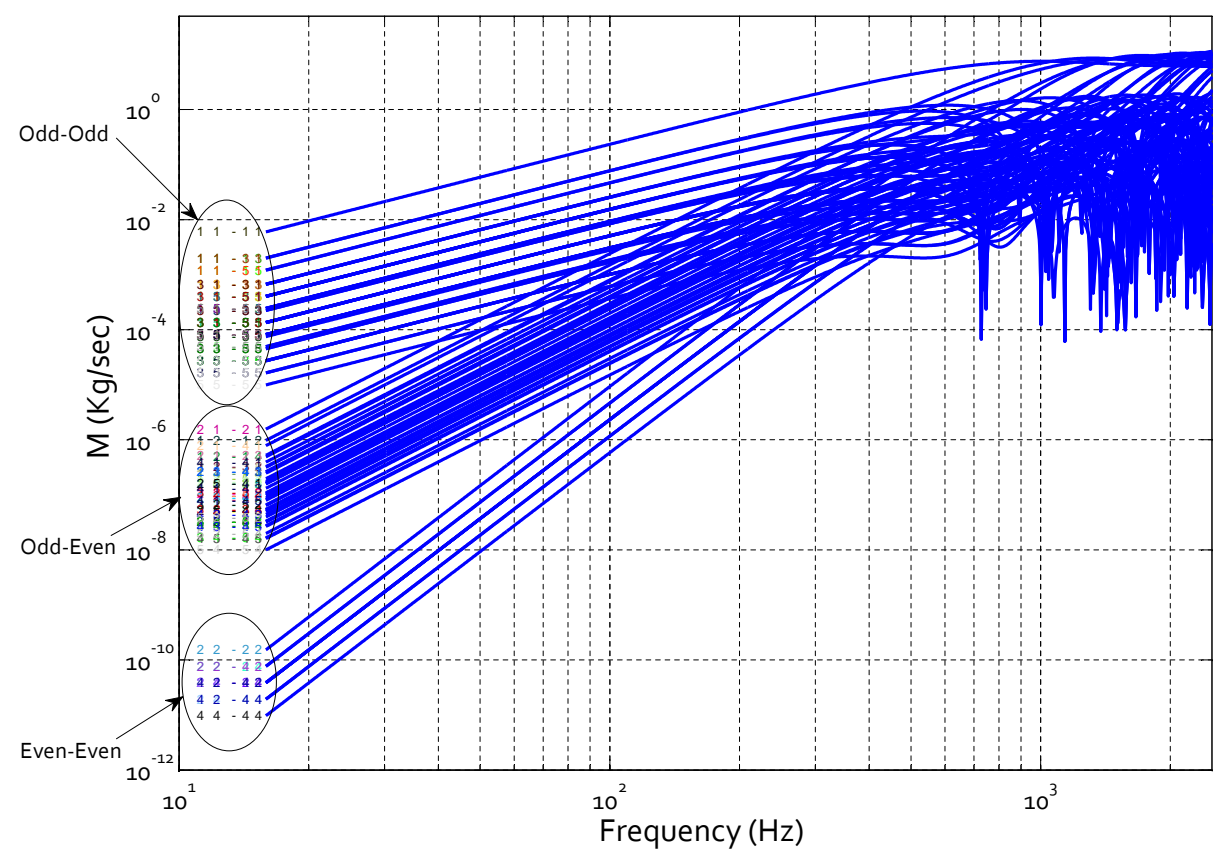

FigURE 4 - Radiation Resistance matrix of structural modes

vicinity of the first mode. This effect is also observable at frequencies situated between the resonances of the other modes, where the first mode produces significant modal contributions to sound power (figure 4). One can note the very good control efficiency on mode three, which was unheeded by usual controls. Consequently the fourth mode four, which is near the third mode, almost disappears and the frequency where the TL is minimum is translated to the right.

Simulations with a higher number of modes were performed to evaluate the influence of a truncated model used by the observer. Figure 6 shows the Transmission Loss in a wider frequency range up to $2500 \mathrm{~Hz}$. From $1200 \mathrm{~Hz}$ to $2500 \mathrm{~Hz}$, one can noticed that Mass-Damping control generates a small spillover whereas other controls do not affect the isolation. The eventual spillover increasing the motion of the incident plate is filtered by the air cavity coupling the two panels. The Transmission Loss remains higher than $80 \mathrm{~dB}$.

The magnitude of the command signal, in the case of a $96 \mathrm{~dB}$ diffuse field disturbance, is showed in Figure 7. The acceleration control voltages tend to be higher than those required for the vibroacoustic controller (damping on radiating modes). It can be assumed that transmission losses are significantly enhanced between $100 \mathrm{~Hz}$ and $200 \mathrm{~Hz}$, due to the increase in control voltage of actuator 2, at frequencies between the first mode resonances (in-phase and out-of-phase). However, these voltage increases are small and clearly feasible in practice. 


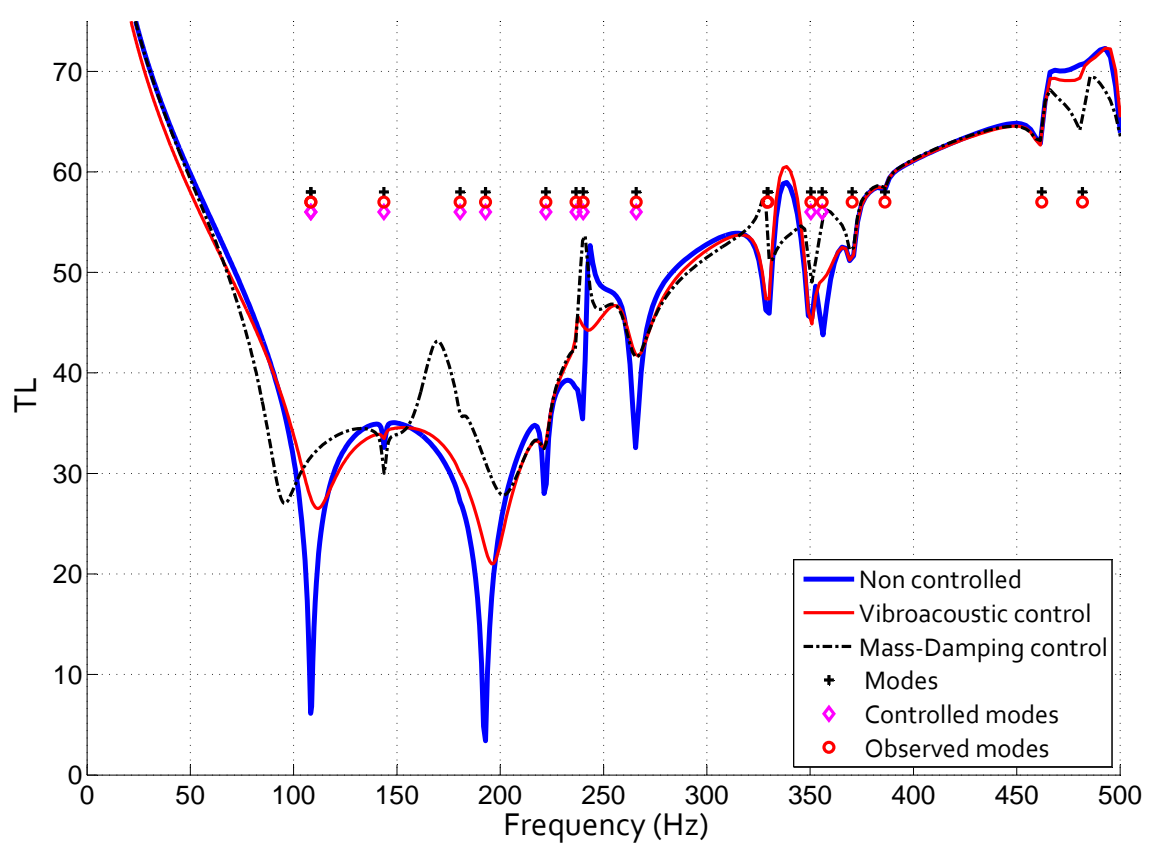

Figure 5 - Double panel Transmission Loss with and without control in the case of a $96 \mathrm{~dB}$ diffuse field excitation from 20 to $500 \mathrm{~Hz}$

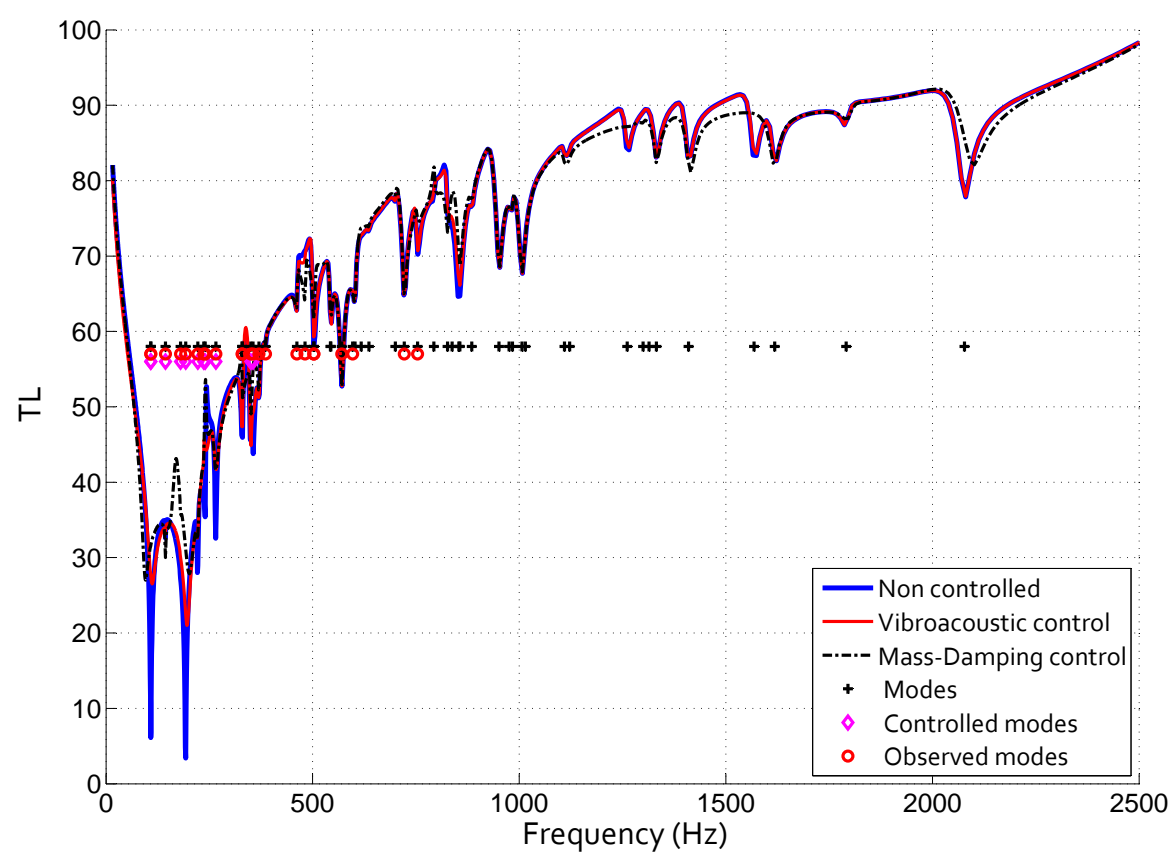

Figure 6 - Double panel Transmission Loss with and without control in the case of a $96 \mathrm{~dB}$ diffuse field excitation from 20 to $2500 \mathrm{~Hz}$

\section{Conclusion}

A modal mass - damping active control is proposed to reduce the transmission of sound through finite double panels. This control has been developed in the framework 


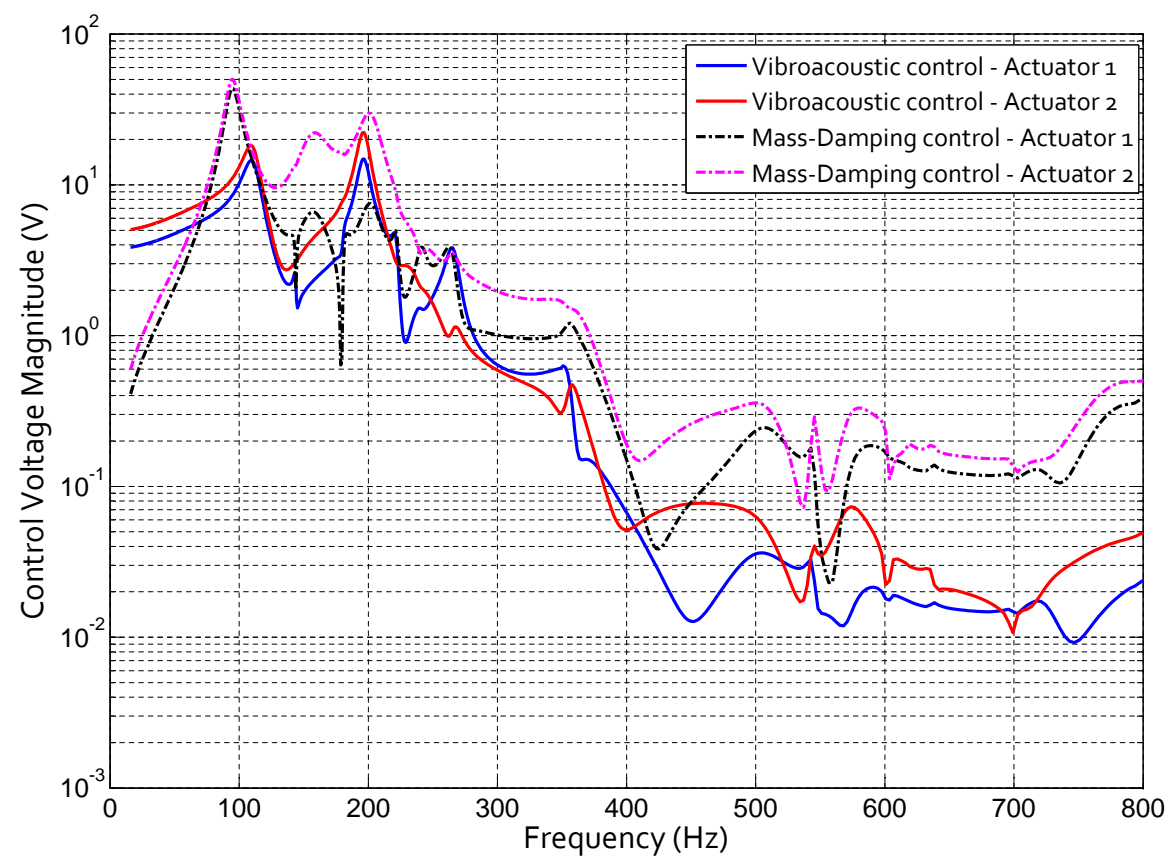

FiguRE 7 - Magnitude of the actuator control voltages for different controllers

of an acoustic system designed to control sound transmission at low frequencies. A state space model has been constructed, including the equations of the structure, the actuators, the acoustic excitation and the acoustic radiation. The implementation of various active controls is also described. It is shown that modal-mass-damping control reduces the peaks at the resonance frequency through damping, in the same way as a radiation filters controller, and also improves the panel's general transmission loss behavior, due to the influence of an additional virtual mass. Contrary to the influence of damping, the mass effect is not restricted to frequencies in the vicinity of the resonance frequency of the controlled modes. Indeed, the addition of an active modal mass lowers the eigenfrequency of the mass-controlled mode, and reduces its velocity at frequencies beyond the resonance frequency. All of these phenomena lead to a slight increase in TL, for the case of small control voltage modifications. In comparison to vibroacoustic control, the voltages of this new controller are not very high, thus indicating that our approach is feasible. Real experiments will raise a whole set of new problems, in particular that of determining the accurate structural model needed by the controller.

\section{Références}

N. Alujevic and P. Gardonio. Decentralized feedback control systems in double panel. In Proceeding of ISMA 2006, Katholieke Universiteit Leuven (Belgium), 2006.

C. Bao and J. Pan. Experimental study of different approaches for active control of sound transmission through double walls. Journal of the Acoustical Society of America, 102 (3) :1664-1670, 1997.

W.T. Baumann. Active suppression of acoustic radiation from impulsively excited structures. Journal of the Acoustical Society of America, 90(6) :3202-3208, 1991.

W.T. Baumann, Fu-Sheng. Ho, and Harry. H. Robertshaw. Active structural acoustic control of broadband disturbances. Journal of the Acoustical Society of America, 92 (4) :1998-2005, 1992. 
B. Bingham, M. J. Attalla, and N. W. Hagood. Model-based feedback control of acoustic radiation from vibrating structures by means of structural control. Journal of Sound and Vibration, 244(5):761-778, 2001.

W. Dehandschutter, K. Henrouille, J. Swevers, and P. Sas. Model-based feedback control of acoustic radiation from vibrating structures by means of structural control. Flow, Turbulance and combustion, 108(2) :239-254, 1999.

E.K. Dimitriadis and C.R. Fuller. Investigation on active control of sound radiation from a panel using piezoelectric actuators. American Institute of Aeronautics and Astronaumics Journal, 29(11) :1771-1777, 1991.

S.J. Elliott and M.E. Johnson. Radiation modes and the active control of sound power. Journal of the Acoustical Society of America, 94(4) :2194-2204, 1993.

Frank Fahy. Sound and structural vibration - Radiation, Transmission and Response. Academic Press inc (London) LTD, 24 - 28 Oval Road - London NW1 7DX, 1985.

A. Francois, P. De Man, and A. Preumont. Piezoelectric array sensing of volume displacement : A hardware demonstration. Journal of Sound and Vibration, 244(3) : 395-405, 2001.

L. Gaudiller and J. Der Hagopian. Active control of flexible structures using a minimum of components. Journal of Sound and Vibration, 193(3) :713-741, 1996.

L. Gaudiller and F. Matichard. A nonlinear method for improving active control efficiency of smart structures subjected to rigid body motions. IEEE/ASME/Transaction of Mechatronics, 12(5) :542-548, 2007.

A. Jakob, M. Moser, and I. Yuksek. Active control of double-glazed windows part i : Feedforward control. Applied Acoustics, 64 :163-182, 2003a.

A. Jakob, M. Moser, and I. Yuksek. Active control of double-glazed windows part 2 : Feedback control. Applied Acoustics, 64 :183-196, 2003b.

O. E. Kaiser, S. J. Pietrzko, and M. Morari. Feedback control of sound transmission through a double glazed window. Journal of Sound and Vibration, 263 :775-795, 2003.

V. Lhuillier, L. Gaudiller, C. Pézerat, and S. Chesné. Improvement of transmission loss using active control with virtual modal mass. Advances in Acoustics and Vibration, Vol $2008: 1-9,2008$.

D.G. Luenberger. Observers for multivariable systems. I.E.E.E., Transactions on Automatic Control, 11 :190-197, 1966.

Q. Mao and S. Pietrzko. Control of sound transmission through double wall partitions using optimally tuned helmholtz resonators. Acta Acustica united with Acustica, 91 : 723-731, 2005.

J. M. Mason and F. J. Fahy. The use of acoustically tuned resonators to improve the sound transmission loss of the double-partition partitions.

F. Matichard and L. Gaudiller. Improvement of potential energetic exhange using non linear control. In IEEE-ASME 2005, AIM - Advanced Intelligent Mechatronics, pages 807-812, Monterey, California, USA, July 24-28 2005.

Bouvet P. Contrôle actif de la transmission acoustique au travers de cloisons doubles. PhD thesis, Université d'Aix-Marseille 2, Marseille, FRANCE, 1993.

S.J Pietrzko and Q. Mao. New results in active and passive control of sound transmission through double wall structures. Aerospace science and technology, 2008. 
A. Preumont, A. Francois, P. De Man, and K. Henrouille. Distributed sensors with piezoelectic films in design of spatial filters for structural control. Journal of Sound and Vibration, 282(2005) :701-712, 2005.

André Preumont. Vibration of Active Structures - An Introduction - 2nd Edition. Kluwer Academic Publishers, P.O Box 17, 3300 AA Dordrecht, The Netherlands, 2002.

M. H. Richardson and D. L. Formanti. Parameter estimation from frequency response measurements using rational fraction polynomials. November 1982.

Louis Roussos. Noise transmission loss of a rectangular plate in a infinite baffle. NASA TP-2398, 1985.

P. Sas, C. Bao, F. Augusztinovicz, and W. Desmet. Active control of sound transmission through a double panel partition. Journal of Sound and Vibration, 180(4) :609-625, 2005 .

T.C. Sors and S.J. Elliott. Volume velocity estimation with accelerometer arrays for active structural acoustic control. Journal of Sound and Vibration, 258(5) :867-883, 2002 .

$\mathrm{R}$ Vaicaitis. Study of noise transmission through double wall aircraft windows. NASA CONTRACTOR REPORT 172182, 1983. 